\title{
Imaging findings on contrast-enhanced ultrasound, magnetic resonance imaging and hybrid positron emission tomography in Takayasu arteritis: a case report
}

\author{
Yonggeng Goh ${ }^{1}$, Eric YS Ting ${ }^{1}$, Jeevesh Kapur ${ }^{1}$, Melati Dewi ${ }^{1}$, Arvind K Sinha ${ }^{2}$, Vijay K \\ Sharma $^{3}$
}

${ }^{1}$ Department of Diagnostic Imaging, ${ }^{2}$ Department of Nuclear Medicine, ${ }^{3}$ Division of Neurology, National University Hospital, Singapore and Yong Loo Lin School of Medicine, National University of Singapore, Singapore

\begin{abstract}
This case report demonstrates the potential of contrast-enhanced ultrasound (CEUS) in diagnosing active arterial wall inflammation in a symptomatic patient with Takayasu arteritis (TA). To our knowledge, this is the first case which demonstrates pictorial correlation of arterial wall neovascularity on CEUS with mural edema on magnetic resonance imaging and metabolic activity on positron emission tomography - computed tomography in the same patient. As TA is a chronic disease which requires long-term follow-up, CEUS could be the potential imaging modality of choice as it is radiation-free, non-nephrotoxic and easily available.
\end{abstract}

Keywords: contrast-enhanced ultrasound; neovascularization; Takayasu arteritis; magnetic resonance imaging; hybrid positron emission tomography

\section{Introduction}

Takayasu arteritis (TA) is a granulomatous vasculitis of unknown etiology, which results in stenosis, occlusion or aneurysms in large arteries [1]. It commonly affects the pulmonary arteries, coronary arteries and proximal segments of various branches of the thoracic aorta. Imaging is crucial in diagnosis and also in follow-up of disease activity after treatment. However, there is currently no international guidelines available which advise on the

Received 10.08.2019 Accepted 13.11.2019

Med Ultrason

2020, Vol. 22, No 4, 485-487

Corresponding author: Dr. Vijay Sharma, MD

NUHS Tower Block, Level 10,

1E Kent Ridge Road, 119228 Singapore

Phone: +65-9138 9555

E-mail: Vijay_Kumar_SHARMA@nuhs.edu.sg type and usage of imaging modalities due to the lack of consistent and specific indices in measurement of disease activity [2].

Contrast-enhanced ultrasound (CEUS) has emerged as a potential imaging tool for arterial wall imaging as it is able to demonstrate arterial wall neovascularization, a marker of active wall inflammation [3]. The usage of CEUS is unfortunately rarely described in literature [4,5] with many other studies favoring the use of magnetic resonance imaging (MRI) and ${ }^{18}$ FDG (Flurodeoxyglucose) positron emission tomography - computed tomography (PET-CT) [6-10], due to the functional and metabolic information of the arterial wall provided.

Hence, this case report aims to demonstrate the potential of CEUS in diagnosing active arterial wall inflammation in TA with pictorial correlation with other imaging modalities such as MRI and PET-CT in the same patient. 


\section{Case report}

A 52-year-old Chinese man with no past medical history presented with recurrent episodes of near syncope and right sided weakness. On clinical examination, a bruit was audible on the left side of his neck. Cervical duplex ultrasonography (US) was performed and revealed diffuse wall-thickening of both common carotid arteries (CCA), worse on the left with an eccentric wallthickening component resulting in mild stenosis (fig 1a). CEUS was performed with iU22 xMATRIX (Philips) at a low mechanical index of 0.10 . Single bolus of $2.5 \mathrm{ml}$ Sonovue (Braco, Milan) was injected followed by $5 \mathrm{ml}$ of saline flush in a peripheral intravenous line. Moderate neovascularization of the arterial wall thickening on the left CCA was found, raising a suspicion of underlying active arterial wall inflammation (fig 1b,c). High resolution MRI of the carotid arteries demonstrated bilateral CCA wall thickening with eccentric mural edema on the left (fig 1d), correlating with the CEUS abnormality. PET/ CT showed circumferential ${ }^{18} \mathrm{FDG}$ uptake in the arterial walls of the great vessels such as the aorta, CCA and subclavian arteries, affirming the diagnosis of Takayasu arteritis (fig 1e). In particular, there was moderate-severe FDG uptake noted in the left CCA, in keeping with active disease accounting for the patient's symptoms. Interestingly, the patient had an unremarkable blood test with no raised inflammatory markers (i.e. Total White Cell Count $=7.700 / \mathrm{mm}^{3}$, Erythrocyte Sedimentation Rate $=8 / \mathrm{h}, \mathrm{C}$ reactive protein $=0.8 \mathrm{mg} / \mathrm{dl}$ ). This is in keeping with the known miscorrelation between systemic inflammatory response of blood markers with active inflammatory processes in arterial walls [1]. The patient was treated with corticosteroids and methotrexate and has recovered well with no further symptoms to date.

\section{Discussion}

Imaging in TA is challenging. To date, catheter angiography is considered the gold standard for diagnosis but is unfortunately invasive and non-feasible for repeated follow-ups. Hence, non-invasive modalities such as computed tomography angiography (CTA) and MRI are usually favored and these techniques demonstrate acceptable accuracy in diagnosis and follow-up of disease activity [11]. However, repeated imaging with CTA is also not ideal as it requires the use of ionizing radiation and nephrotoxic contrast agents. On the other hand, MRI is radiation-free but is considerably more expensive than CTA and may not be cost-effective. ${ }^{18}$ FDG PET-CT

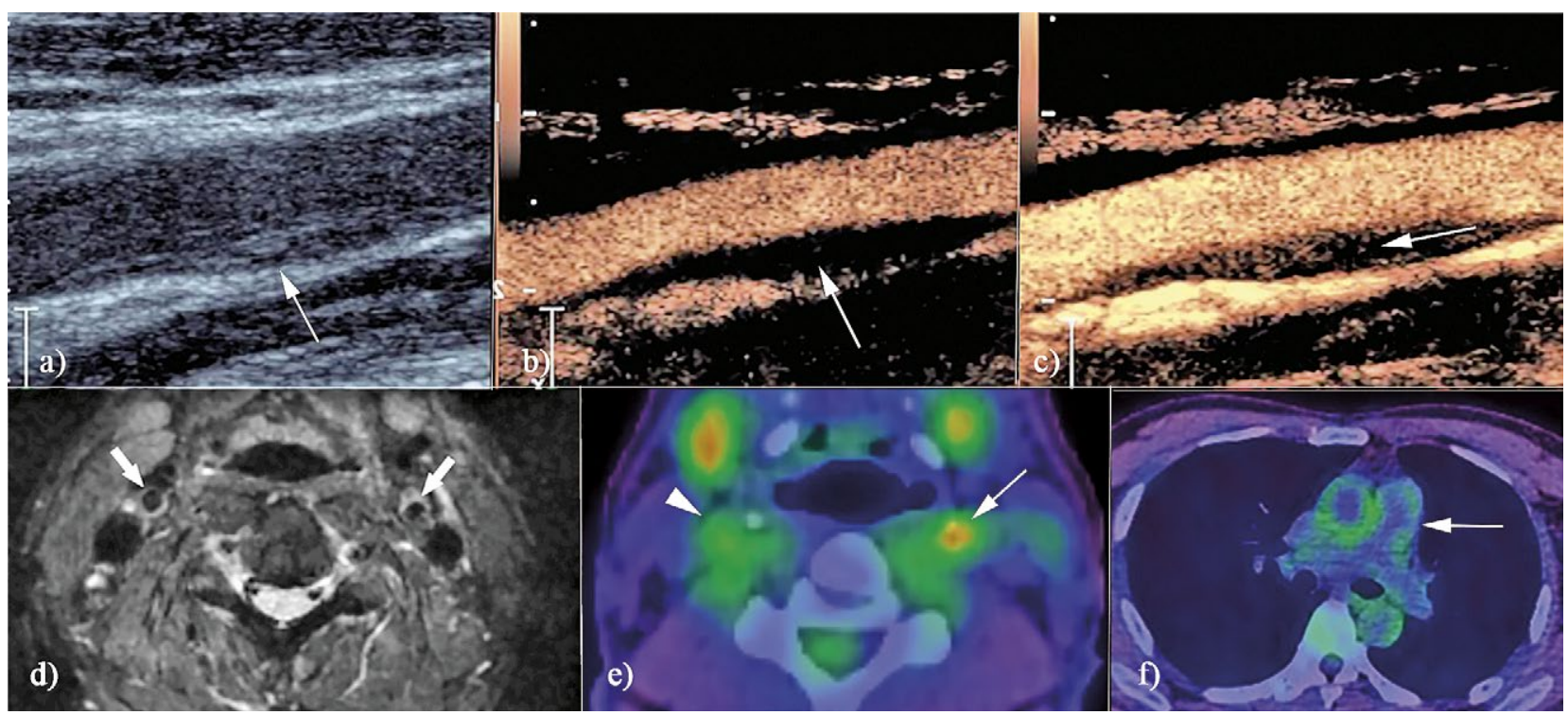

Fig 1. CEUS with dual-screen display of the left CCA in grayscale image (a) and contrast images side-by-side (b and c) shows eccentric segmental wall thickening of the left CCA (arrow). At 5 seconds after Sonovue injection, there was luminal contrast opacification outlining the wall thickening (b). At 10 seconds (c), microbubble uptake was noted within the abnormally thickened CCA wall (arrow), suggestive of pathogenic neovascularization and active inflammation; d) Axial T2-weighted MRI of the carotid arteries shows diffuse hyperintense circumferential wall thickening of both CCAs (arrows). On the left, there is the presence of mural edema; e) metabolic imaging with FDG-18F PET/CT demonstrated increased circumferential FDG avidity in both CCAs, standard uptake value $3.5 \mathrm{gm} / \mathrm{ml}$ in right CCA (arrowhead) and $4.6 \mathrm{~g} / \mathrm{ml}$ in left CCA (arrow); f) There was also increased circumferential uptake seen in the great vessels such as main pulmonary artery (arrow), suggestive of active inflammatory changes. Overall, the imaging findings were suggestive of large vessel arteritis such as Takayasu arteritis. 
has been used increasingly in recent years [12] due to its wide field-of-view and high sensitivity in detection of metabolic activity in arterial walls, but incurs even higher costs and a radiation dose compared with MRI and CTA respectively [13]. There is hence a clinical need for an imaging modality for disease follow-up in TA which is radiation-free and cost-effective.

Our case has showed the utility of CEUS in demonstrating active arterial wall inflammation in TA by visualizing the increased neovascularization in the arterial wall. The use of CEUS can be particularly useful in TA patients with localized wall inflammation [14]. The neovascularization on CEUS also correlated well with mural edema on MRI and ${ }^{18} \mathrm{FDG}$ activity on PET/CT, suggesting that neovascularization on CEUS could be a possible marker in disease follow-up. Nonetheless, this is the first case to the authors' knowledge of an intra-individual correlation of arterial wall imaging across different modalities which demonstrated the anatomical, functional and metabolic aspects of the disease process.

In conclusion, neovascularization of the arterial wall is highly suggestive of underlying active inflammation and its role as a potential index for TA disease activity measurement requires to be validated in studies with larger sample sizes.

\section{References}

1. Kerr GS, Hallahan CW, Giordano J, et al. Takayasu arteritis. Ann Intern Med 1994;120:919-929.

2. Direskeneli H, Aydin SZ, Merkel PA. Assessment of disease activity and progression in Takayasu's arteritis. Clin Exp Rheumatol 2011;29(1 Suppl 64):S86-S91.

3. Herlin B, Baud JM, Chadenat ML, Pico F. Contrast-enhanced ultrasonography in Takayasu arteritis: watching and monitoring the arterial inflammation. BMJ Case Rep 2015;2015:bcr2015211094.

4. Ma LY, Li CL, Ma LL, et al. Value of contrast-enhanced ultrasonography of the carotid artery for evaluating dis- ease activity in Takayasu arteritis. Arthritis Res Ther 2019; 21:24.

5. Wang Y, Wang YH, Tian XP, et al. Contrast-enhanced ultrasound for evaluating arteritis activity in Takayasu arteritis patients. Clin Rheumatol 2019. doi:10.1007/s10067-01904698-9

6. Nastri MV, Baptista LP, Baroni RH, et al. Gadoliniumenhanced three-dimensional MR angiography of Takayasu arteritis. Radiographics 2004;24:773-786.

7. Aluquin VP, Albano SA, Chan F, Sandborg C, Pitlick PT. Magnetic resonance imaging in the diagnosis and follow up of Takayasu's arteritis in children. Ann Rheum Dis 2002;61:526-529.

8. Liu M, Liu W, Li H, Shu X, Tao X, Zhai Z. Evaluation of takayasu arteritis with delayed contrast-enhanced MR imaging by a free-breathing 3D IR turbo FLASH. Medicine (Baltimore) 2017;96:e9284.

9. Slart R, Writing group, Reviewer group, et al. FDG-PET/ $\mathrm{CT}(\mathrm{A})$ imaging in large vessel vasculitis and polymyalgia rheumatica: joint procedural recommendation of the EANM, SNMMI, and the PET Interest Group (PIG), and endorsed by the ASNC. Eur J Nucl Med Mol Imaging 2018;45:1250-1269.

10. Tezuka D, Haraguchi G, Ishihara T, et al. Role of FDG PETCT in Takayasu arteritis: sensitive detection of recurrences. JACC Cardiovasc Imaging 2012;5:422-429.

11. Espigol-Frigole G, Prieto-Gonzalez S, Alba MA, et al. Advances in the diagnosis of large vessel vasculitis. Rheum Dis Clin North Am 2015;41:125-140.

12. Santhosh S, Mittal BR, Gayana S, Bhattacharya A, Sharma A, Jain S. F-18 FDG PET/CT in the evaluation of Takayasu arteritis: an experience from the tropics. J Nucl Cardiol 2014;21:993-1000.

13. Fuchs M, Briel M, Daikeler T, et al. The impact of $18 \mathrm{~F}-$ FDG PET on the management of patients with suspected large vessel vasculitis. Eur J Nucl Med Mol Imaging 2012;39:344-353.

14. Mavrogeni S, Dimitroulas T, Chatziioannou SN, Kitas G. The role of multimodality imaging in the evaluation of Takayasu arteritis. Semin Arthritis Rheum 2013;42:401412. 